\title{
High and low beam energy imaging: complementarity in a monochromated XHR SEM
}

\author{
L.Y. Roussel*, I. Gestmann*, M. Darus**, F. Morrissey* and D.J. Stokes* \\ * FEI Company, Building AAE, PO Box 80066, 5600 KA Eindhoven, The Netherlands \\ ** FEI Company, 5350 NE Dawson Creek Drive, Hillsboro, OR 97124, USA
}

With traditional scanning electron microscopes (SEM), the characterization of nanostructures has always resulted in a trade-off between spatial resolution and surface sensitivity. At higher beam energies (typically $15 \mathrm{kV}$ and above), nanometer resolution is easily achieved, while the actual information collected results from both the surface and the depth of the sample. The lower the beam energy, the more surface sensitive the collected information becomes, yet at the price of spatial resolution. This is mainly because, at low beam energy, conventional electron sources and optics are not able to compensate for what degrades the spot size most: chromatic aberration, due to energy spread of the electrons emitted by the source.

Modern ultra-high resolution (UHR) field-emission SEMs are now able to narrow the spatial resolution gap between beam energies of 1 and $30 \mathrm{kV}$, using refined optics and electron sources with a reasonable energy spread. At $1 \mathrm{kV}$, the spatial resolution gets to the range of $1.5 \mathrm{~nm}$, but the penetration of electrons in the material is still on the order of a few tens of nanometers. This is why UHR SEMs are now routinely offering the possibility to use beam landing energies of just $100 \mathrm{~V}$ and below for true surface imaging, using techniques such as pre- or post-deceleration [1,2]. This, unfortunately, comes again at the expense of spatial resolution since, although the energy spread of primary electrons is improved by maintaining a higher beam energy up to a late stage in the process, some chromatic aberration remains. The long awaited next step [3] only happened very recently, with the introduction of the first monochromated extreme high resolution (XHR) SEM [4]. Not only does the XHR SEM allow to achieve sub-nanometer spatial resolution using a $1 \mathrm{kV}$ primary beam energy on bulk specimens, but also $1.5 \mathrm{~nm}$ spatial resolution is demonstrated at a beam landing energy of $200 \mathrm{~V}$. In the latter case, an interaction volume shrinks down to only a few nanometers in depth.

In this study, an FEI Magellan 400 XHR SEM is used to explore high resolution at high and very low beam energies on bulk and thin samples, as well as the complementary information that becomes available with the XHR SEM. It is equipped with a newly integrated high performance STEM detector capable of collecting bright field, dark field and high angle signals after the primary electron beam has passed through a thin specimen. Coupling low and very low beam energy SEM to $30 \mathrm{kV}$ STEM-in-SEM means that highly surface sensitive and volumetric data is rapidly obtained at the very nanometer level, from the same region of a given specimen.

References

[1] I. Müllerova and L. Frank, Modern Research and Educational Topics in Microscopy, Ed. Méndez-Vilas and Diaz (2007) 795

[2] H. Jaksch and J. P. Martin, Fresenius' Journal of Analytical Chemistry, Vol. 353, Numbers 3-4 (1995) 378

[3] E.D. Boyes, Microsc Microanal 11(supp12) (2005) 2112 
[4] R. J. Young et al, Microscopy Today, Vol 16, Number 4 (2008) 24

Figures 1 and 2 illustrate high resolution and high surface sensitivity using very low beam landing energy on a deprocessed integrated circuit, respectively $200 \mathrm{~V}$ and $50 \mathrm{~V}$ in secondary electron mode (sample courtesy of ST Microelectronics Grenoble and Malta). Figure 3 shows a carbon nanotube obtained by STEM-in-SEM in bright field mode at $30 \mathrm{kV}$ (outer diameter roughly $75 \mathrm{~nm}$, inner diameter roughly $15 \mathrm{~nm}$ ), with nicely contrasted volumetric information. A comparable nanotube, this time imaged using a landing energy of $200 \mathrm{~V}$ in secondary electron mode (figure 4), shows detailed surface information at about the same magnification as figure 3 .

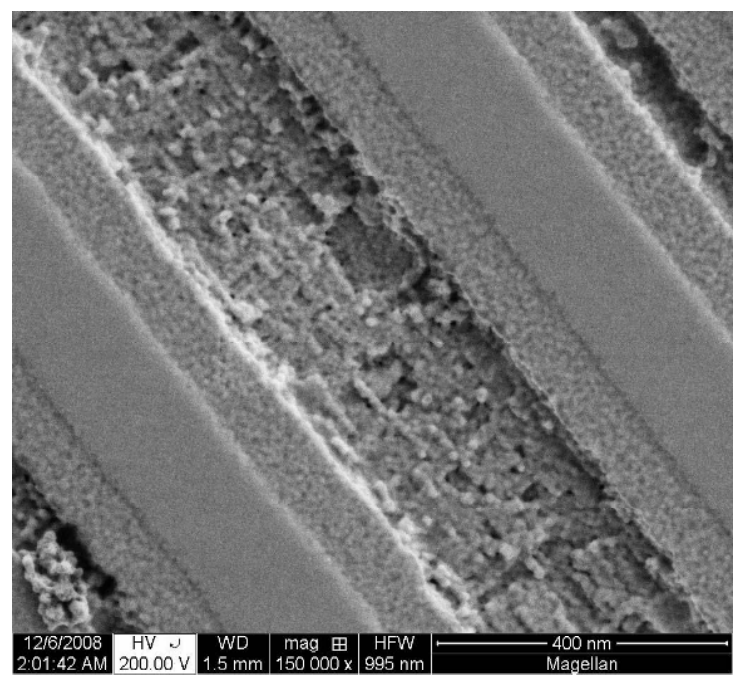

Figure 1

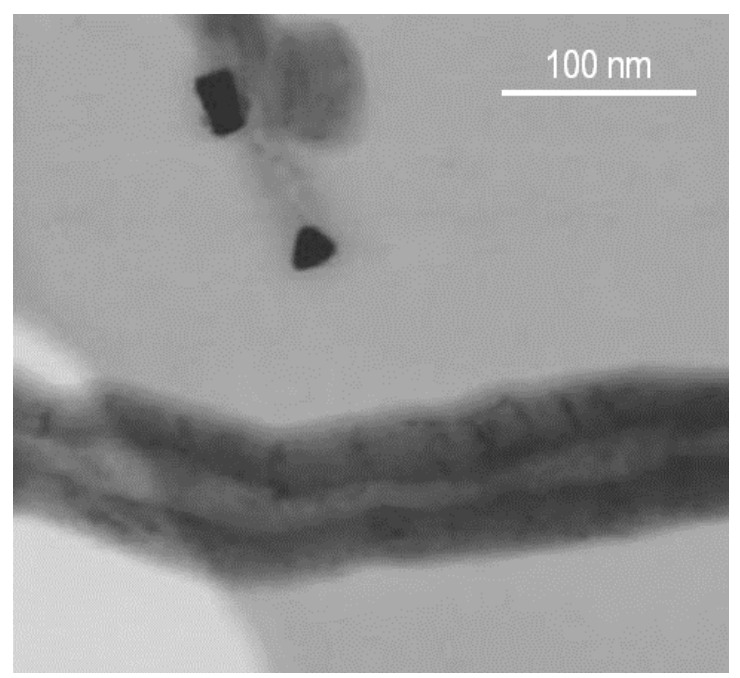

Figure 3

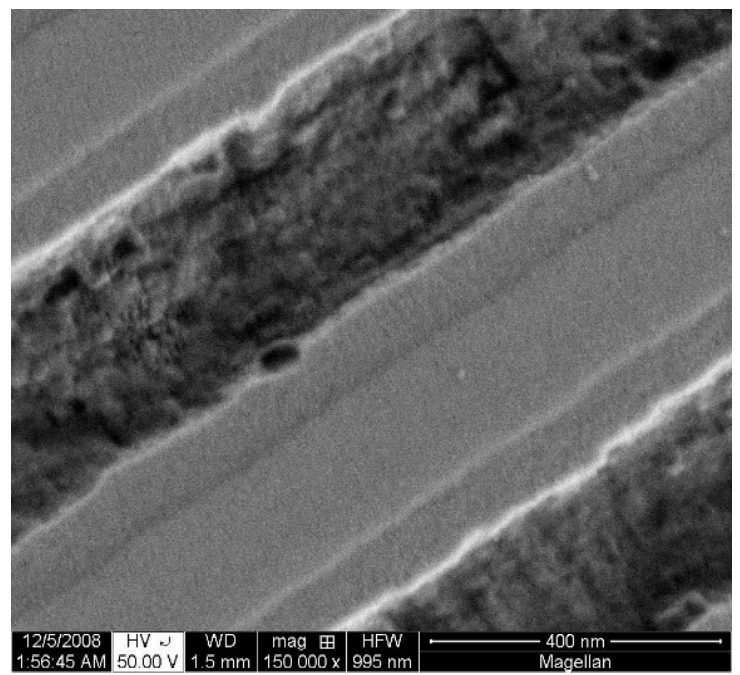

Figure 2

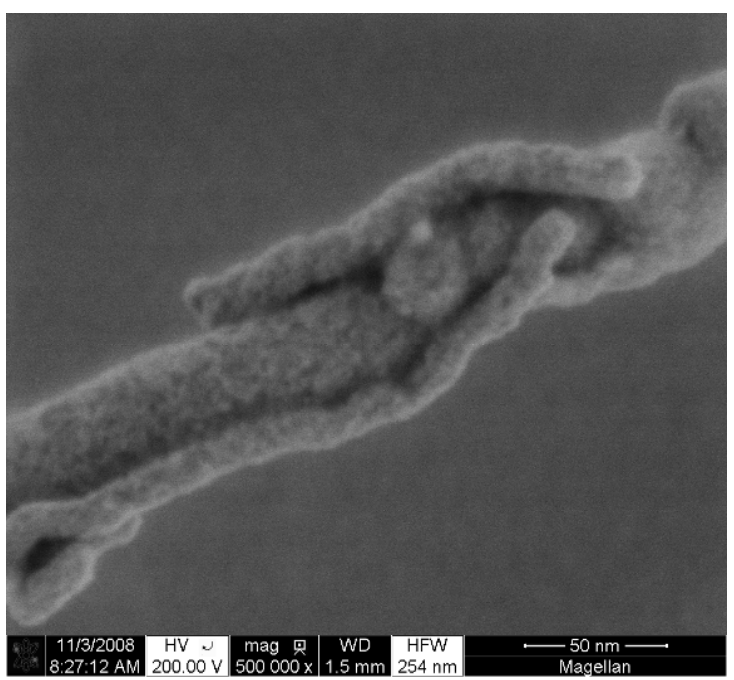

Figure 4 